\title{
Deficient Lymphokine Production of Newborn Lymphocytes
}

\author{
HARLAND S. WINTER, ${ }^{(26)}$ SHERRIE E. GARD, THOMAS J. FISCHER ${ }^{(27)}$ YVONNE J. BRYSON, \\ AND E. RICHARD STIEHM ${ }^{(28)}$ \\ Divisions of Immunology/Allergy and Infectious Diseases, Department of Pediatrics, Center for the Health Sciences, \\ University of California School of Medicine, Los Angeles, California, USA
}

\begin{abstract}
Summary
Lymphokine production by newborn lymphocytes was assessed by measuring migration inhibition factor (MIF) and leukocyte inhibition factor (LIF) of isolated mononuclear cells from cord blood, 1-7-days-old newborns, and adult controls. Ficoll-Hypaque separated mononuclear cells were stimulated with phytohemagglutinin (PHA) or allogeneic lymphocytes in a mixed leukocyte culture (MLC), and the supernatants were harvested at optimal times for lymphokine assays. Thymidine incorporation into DNA was also assayed to calculate a proliferative index. MIF was assessed by the inhibition of adult mononuclear phagocyte cell migration under agarose; LIF was assessed by polymorphonuclear cell migration under agarose. Although the proliferative responses of cord and newborn cells are equivalent or greater than those of adult controls, the PHA-induced MIF production in cord blood and newborn lymphocytes was only $46 \%$ and $12.5 \%$ respectively of mean adult levels; MLC-induced MIF production was $44 \%$ and $7 \%$, respectively of mean adult levels. PHA-induced LIF production in cord blood was $27 \%$ of adult levels. These differences are only appreciated if dilutions of the supernatants are assayed. Simultaneous assay of MIF and LIF production in dilution of supernatants from adult lymphocytes showed higher LIF activity, whereas in cord lymphocytes MIF activity was greater than LIF activity. This further emphasizes the non-identity of MIF and LIF.

These results indicate another abnormality of $T$ cellular immunity in newborns not detected by $T$-cell enumeration or proliferative responses and parallels other defects in specialized $T$ cell function such as cytotoxicity and immune interferon production.
\end{abstract}

\section{Abbreviations}

CPM, counts per minute

FCS, fetal calf serum

IF, inhibition factor

LIF, leukocyte inhibition factor

MIF, migration inhibition factor

MLC, mixed leukocyte culture

MN, mononuclear

PHA, phytohemagglutinin

PPD, purified protein derivative

Despite normal thymic size and function, normal $\mathrm{T}$ cell numbers and subsets, and vigorous proliferative responses to mitogens, cellular immunity in the newborn human is functionally deficient. This is suggested by the newborns' enhanced susceptibility to viral infection $(4,17)$, diminished delayed cutaneous hypersensitivity reactions $(2,24)$, and selective abnormalities (when compared to adults) of in vitro measures of cellular immunity including impaired proliferative responses to ubiquitous antigens (12), depressed lymphokine production (7), variably diminished cytotoxic reactions $(1,5,14,21,22,23)$ and excessive $T$ suppressor activity.

Lymphokine deficiency in the presence of normal proliferative responses is characteristic of a number of illnesses associated with anergy, and was first observed in the newborn by Eife et al. (7) in their studies of lymphotoxin production in cord blood. We recently reported deficient immune interferon production despite normal classical interferon production, suggesting selective lymphokine abnormalities among newborns (3). Utilizing many of the same supernatants generated during the interferon studies, we measured MIF and LIF activity in cord blood lymphocytes and newborn lymphocytes after activation with PHA or allogeneic lymphocytes in a MLC.

A new adaptation of the agarose chemotactic assay (10) was used to measure MIF in the supernatant of cultured cells and the Clausen modification of the polymorphonuclear leukocyte migration under agarose assay was used to measure LIF. Timed samples were obtained to determine the kinetics of lymphokine production. We found that after serial dilutions of the supernatants there were significant differences in both MIF and LIF production among newborn, cord, and adult cells.

\section{MATERIALS AND METHODS}

Cell separation. After obtaining parental permission in accordance with the regulations of the UCLA Human Subject Protection Committee, peripheral blood samples $(1 \mathrm{ml} / \mathrm{kg})$ were obtained with a scalp vein needle and plastic syringe from the antecubital vein of 29 healthy neonates less than 7 days of age. Thirty-three healthy adult volunteers were also studied. Blood was heparinized (lipo-Hepin) to a final concentration of 20 units $/ \mathrm{ml}$. Thirty-three umbilical cord bloods were obtained and heparinized in an identical fashion at the time of delivery. Mononuclear cells were separated by differential separation using Ficoll-Hypaque density gradients (specific gravity, 1.077-1.084) (11). Approximately $70 \%$ of the cells obtained were lymphocytes and $30 \%$ were MN phagocytes. After three washings with Hank's balanced salt solution, the cell pellet was resuspended in RPMI 1640 supplemented with Pen-Strept, L-glutamine and HEPES buffer and diluted to final concentration $10^{6}$ cells $/ \mathrm{ml}$.

Phytohemagglutinin stimulation. The separated mononuclear cells (final concentration, $2 \times 10^{5} / .2 \mathrm{ml}$ ) were cultured in microtiter plates with PHA (PHA-M, DIFCO Catalog \#0528-57), final concentration $1: 40$ in RPMI 1640 with $10 \%$ pooled heat-inactivated $\mathrm{O}$ positive human serum. Preliminary studies indicated that this concentration of PHA gave optimal transformation. Cultures without PHA and without cells were established for controls. All cultures and controls were established in triplicate and incubated at $37^{\circ} \mathrm{C}$ in a $5 \% \mathrm{CO}_{2}$ humidified atmosphere. The stimulation indices were determined by pulsing one set of cultured cells at 72 h with $2 \mu \mathrm{Ci}$ of tritiated thymidine, incubating for 16 more hours, harvesting the cells on filter paper discs, determining the CPM of the cell pellets, and calculating the ratio of CPM in the PHAstimulated cell cultures to the CPM in unstimulated cultures. Larger cultures ( $1-2 \mathrm{ml}$ ) with identical cell-PHA ratios were established simultaneously in $12 \times 75 \mathrm{~mm}$ tubes (20) and terminated at $48 \mathrm{~h}$ (or other predetermined time) for lymphokine analysis. The supernatants were separated by centrifugation at $450 \times g$ for $10 \mathrm{~min}$ and stored at $-20^{\circ} \mathrm{C}$ until assayed for MIF and LIF. 
$M L C$ stimulation. The responder separated mononuclear cells were washed once in Medium 199 with $1 \%$ AB plasma, and resuspended in Medium 199 with 20\% AB Plasma. Stimulator cells were prepared in an identical fashion and irradiated with $4000 \mathrm{R}$ in a Cesium irradiator. Responder cells were incubated in triplicate with autologous fresh adult, or irradiated frozen pooled mononuclear cells. The latter were obtained from six normal adult donors, separated, pooled, frozen in aliquots in liquid nitrogen, and thawed just before use. Suspensions of stimulator and responder cells ( $1: 1$ ratio) were cultured according to the semi-micro MLC procedure of Sengar and Terasaki (20). One set of cultures was pulsed with tritiated thymidine at day 4 and the cells harvested $16 \mathrm{~h}$ later for determination of stimulation indices. The supernatants of the other cultures were harvested daily for 5 days or only on day 5 , and frozen at $-20^{\circ} \mathrm{C}$ until tested for MIF. The stimulation index of the MLC is the ratio of the CPM of the responder lymphocytes stimulated by the lymphocyte pool and of the CPM of responder cells stimulated by autologous cells.

MIF Assay. MIF in the leukocyte supernatants was assayed by a modification of the agarose MN phagocyte chemotaxis assay described by Cutler (6) and Nelson et al. (16). Klein et al. (10) showed that this technique was of value in assaying MN chemotaxis using a three well system with the cell suspension in the center well. Ten lambda of normal adult $\mathrm{MN}\left(10^{7} / \mathrm{ml}\right)$ cells are placed in the center well. Chemotactic factor (zymosan-treated human serum) is placed in one peripheral well and $0.15 \mathrm{~N} \mathrm{NaCl}$ is placed in the other well. Supernatants from control cultures that have not been activated are used as controls. Migrating cells are counted after $16 \mathrm{~h}$ as previously described (10). The cell migration is calculated as the number of cells migrating toward the chemotactic factor (directed migration) minus the number of cells migrating toward the saline (random migration). MIF activity is expressed as the \% inhibition of chemotaxis, calculated by determining the ratio of the cell migration with activated supernatant and multiplying by 100 . All assays are done in triplicate and averaged.

$L I F$ assay. Fifty milliliters of a $2 \%$ solution of agarose (Fisherelectrophoresis grade) were mixed with a solution of 2 x Med 199 with $20 \% \mathrm{FCS}, 1 \%$ penicillin-streptomycin and L-glutamine and buffered with $\mathrm{NaHCO}_{3}$. Four milliliters of agarose-medium solution were pipetted into $80 \times 9 \mathrm{~mm}$ sterile petri dishes (Falcon 1006) and allowed to set at room temperature. The petri dishes were stored at $37^{\circ} \mathrm{C}$ in $5 \% \mathrm{CO}_{2}$ for as long as a wk. Just before use, six evenly spaced, $4 \mathrm{~mm}$ in diameter wells were punched in each plate and the plugs removed by gentle suction.

Polymorphonuclear cells were separated by sedimentation with $15 \%$ Plasmagel and washed three times in sterile $0.15 \mathrm{~N}$ saline, and counted. $5-10 \times 10^{6}$ cells were pipetted into each sterile $6 \mathrm{ml}$ tube and centrifuged at $150 \times g$ for $5 \mathrm{~min}$. The supernatant was aspirated. For each determination $0.01 \mathrm{ml}$ of LIF supernatant fluid was added for each $10^{6}$ cells, then mixed well and incubated for $30 \mathrm{~min}$ at $37^{\circ} \mathrm{C}$ in $5 \% \mathrm{CO}_{2}$. Each sample was resuspended and 15 lambda was pipetted into each of triplicate wells. The petri dishes were incubated $16-20 \mathrm{~h}$ at $37^{\circ} \mathrm{C}$ in $5 \% \mathrm{CO}_{2}$. After the incubation, the cells were fixed with $2 \mathrm{ml}$ of $7 \%$ glutaraldehyde for $20 \mathrm{~min}$, the gel removed and the plates air dried. Two perpendicular diameters were measured for each well with a calibrated eyepiece and used to calculate the area of migration.

Percent migration equals the mean number of PMN in the area exposed to test supernatants divided by the mean number of PMN in the area exposed to control supernatants.

Statistical analysis. Data is expressed at mean \pm standard deviation. Statistics are calculated using the Student's $t$-test.

\section{RESULTS}

PHA-stimulated MIF production. Three sets of newborn, cord, and adult lymphocytes were stimulated by PHA and the supernatants were harvested for 5 days to determine the kinetics of MIF production. In all three groups of lymphocytes, MIF activity was present by $24 \mathrm{~h}$ and maximal by $48 \mathrm{~h}$. MIF activity did not change between 2-5 days in the stimulated cultures; however, one unstimulated cord lymphocyte sample and one unstimulated newborn lymphocyte sample developed MIF activity on day 5, suggesting the occurrence of spontaneous blastogenesis and lymphokine production. Hereafter, all MIF results are those from supernatants harvested after $48 \mathrm{~h}$ in culture.

In Figure 1, spontaneous and directed migration inhibitory

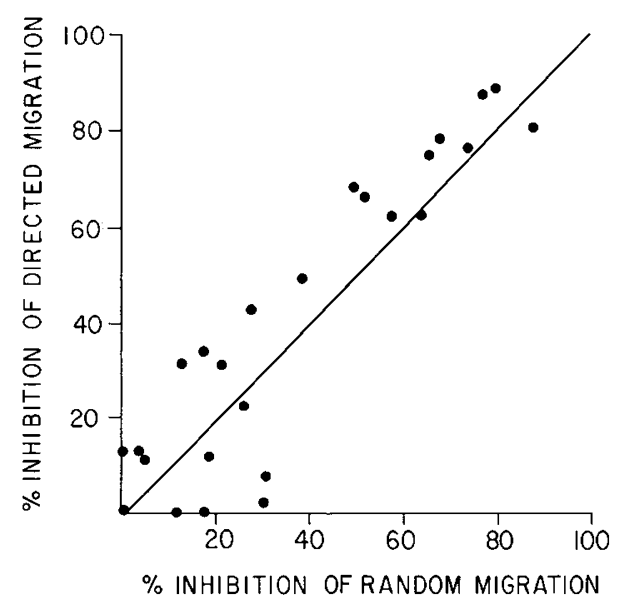

Fig. 1. Correlation of the degree of inhibition of directed and random migration of human monocytes in 25 lymphocyte supernatant dilutions. There was close correlation $(P<0.001)$ between the two. The \% inhibition of migration was calculated by measuring the number of migratory mononuclear cells after exposure to activated supernatants, divided by the number of migrating mononuclear cells not exposed to supernatant $\times 100$.

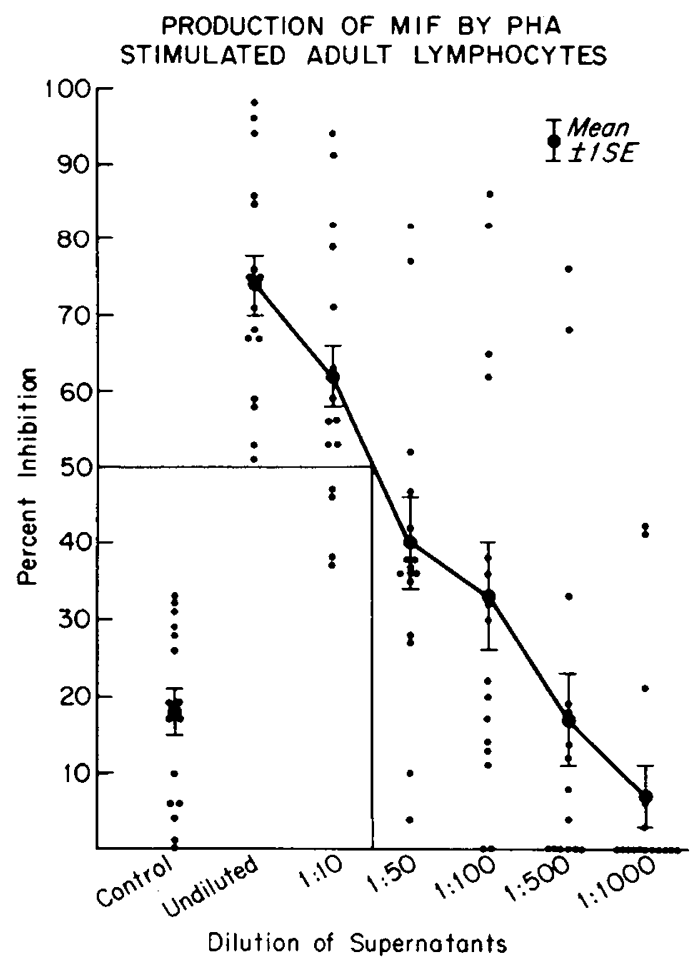

Fig. 2. Production of migration inhibition factor (MIF) by lymphocytes of adults activated with phytohemagglutinin. The MIF activity is expressed as the \% inhibition of migration, calculated by measuring the number of mononuclear cells migrating with and without lymphocyte supernatant $x$ 100. The unstimulated supernatant (at 2 days) has mean MIF activity of $18 \% \pm 3$ (S.E.) compared to the stimulated supernatant of $74 \pm 4$ (S.E.) MIF activity of the activated supernatants remains, even at a $1: 100$ dilution. The dilution giving $50 \%$ inhibition is indicated by the vertical line. 
activity of supernatants were compared. Inhibition of directed movement correlates closely with inhibition of spontaneous migration. No significant inhibitory activity was detected by either method in supernatants of PHA-activated leukocyte cultures of three patients with combined immunodeficiency or Candida-activated leukocyte cultures of three patients with chronic mucocutaneous candidiasis.

As illustrated in Figure 2, the mean MIF activity of supernatants from unstimulated adult lymphocyte cultures was $18 \pm 3 \%$ inhibition. By contrast, the MIF activity of 22 supernatants from adult lymphocyte cultures stimulated with optimal amounts of PHA was $74 \pm 4 \%$ inhibition. As depicted, when dilutions of the supernatants are tested for MIF, activity of $33 \pm 7 \%$ inhibition was still detectable at a dilution of $1: 100$. The horizontal line on Figure 2 indicates the dilution at which $50 \%$ inhibition occurs, a mean $1: 12$ dilution.

The MIF activity of supernatants from unstimulated newborn lymphocyte cultures showed $11 \pm 3 \%$ inhibition; supernatants from 17 PHA-stimulated newborn lymphocytes had a mean MIF activity of $58 \pm 6 \%$ inhibition (Fig. 3). At a dilution of $1: 5$, the MIF activity of the supernatant was $29 \pm 6 \%$. At dilutions of $1: 10$ or greater MIF was not detectable. Fifty percent inhibition occurred at a dilution of $1: 1.5$.

The MIF activity of supernatants from unstimulated cord lymphocyte cultures was $29 \pm 5 \%$ which was significantly greater $(P$ $<0.05)$ than adult or newborn unstimulated supernatants. The mean MIF activity of 24 PHA-stimulated undiluted supernatants was $74 \pm 6 \%$ when diluted 1:10 (Fig. 4). At dilutions of $1: 25$ or greater, the MIF activity was not significantly greater than unstimulated values. Fifty percent inhibition occurred at a dilution of $1: 5.5$. The mean stimulation index of the cord lymphocyte cultures was $198 \pm 42$, not significantly lower than for newborns $(432 \pm$

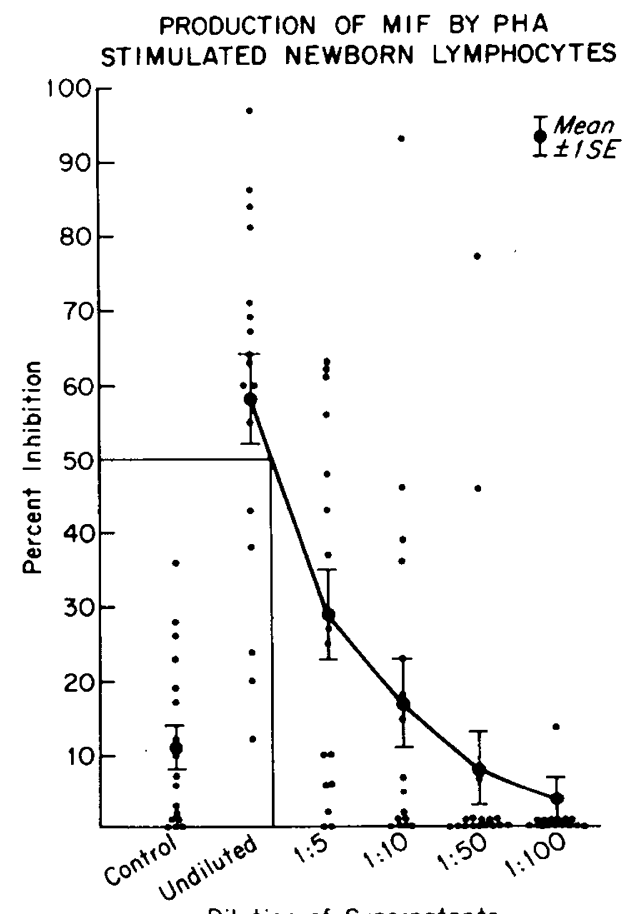

Fig. 3. Production of migration inhibition factor (IF) by newborn lymphocytes activated with phytohemagglutinin. The migration inhibition factor (MIF) activity is expressed as the \% inhibition of monocyte migration calculated by dividing the number of mononuclear cells migrating in the presence of lymphocyte supernatant by the number of mononuclear cells migrating in absence of lymphocyte supernatant $\times 100$. The supernatants from unstimulated newborn lymphocytes had a mean MIF activity of $11 \pm 3 \%$, whereas the undiluted stimulated supernatants had a mean activity of $58 \pm 6 \%$. Fifty percent inhibition was achieved at a dilution of $1: 2$, significantly less than that of adult or cord blood lymphocytes.

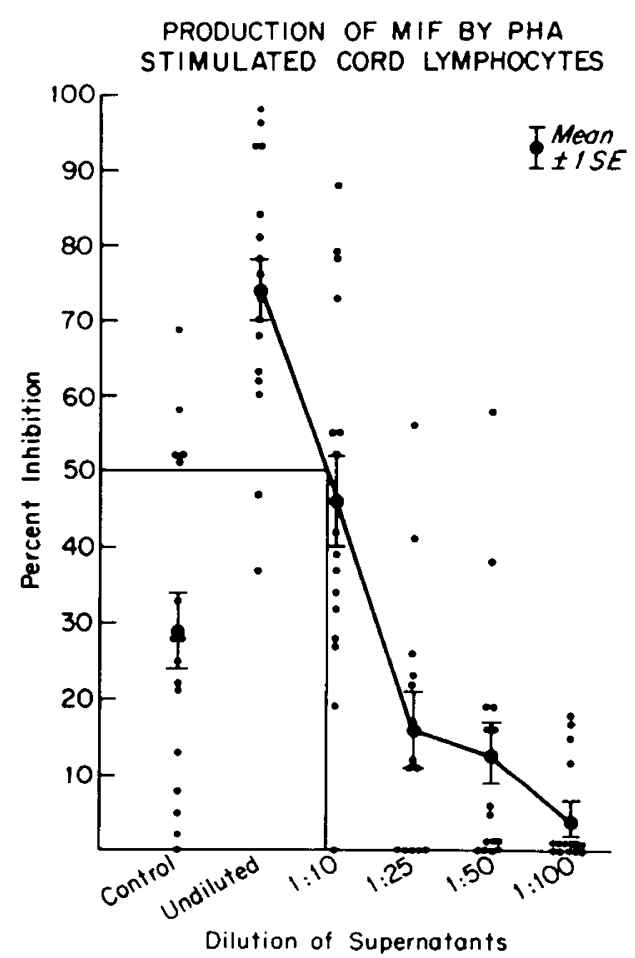

Fig. 4. Production of migration inhibition factor (MIF) by cord blood lymphocytes activated with phytohemagglutinin. The MIF activity is expressed as the \% inhibition of monocyte migration, calculated by dividing the number of mononuclear cells migrating in the presence of lymphocyte supernatant by the number of mononuclear cells migrating in the absence of lymphocyte supernatant $\times 100$. The supernatants from unstimulated cord lymphocytes had a mean MIF activity of $29 \pm 5 \%$ ( \pm S.E.) whereas the undiluted stimulated supernatants had a mean activity of 74 $\pm 4 \%$. Fifty percent inhibition was achieved at a dilution of $1: 8$, significantly less than that of adult lymphocytes.

$150)$, but significantly lower than adult cell cultures $(523 \pm 105)$, because of the high resting values.

A comparison of MIF activity of the three groups of supernatants can be made by noting the dilution that gave 50\% MIF activity (Fig. 5). This was achieved at a 1:12 dilution for adult cell supernatants, at a 1:1.5 dilution of newborn cell supernatants, and at 1:5.5 dilution for the cord cells, indicating that cord lymphocytes have $46 \%$ of the MIF activity of adult cells, and newborn lymphocytes have $12.5 \%$ of the MIF activity of adult cells.

Five pairs of cord and newborn cultures from the same infants were studied for MIF activity. In all five babies, the MIF activity of PHA-stimulated cord blood supernatants was slightly greater (mean $80 \pm 4 \%$ ) than the supernatants from PHA-stimulated newborn lymphocytes (mean $68 \pm 4 \%$ ) obtained 1-7 days after birth. After dilution, the trend for the paired samples reflected the same pattern observed in the unpaired samples; cord lymphocytes produce about four times more MIF than did newborn lymphocytes.

MLC-stimulated MIF production. The supernatants of lymphocyte cultures from two newborns and two adults stimulated by allogeneic pooled cells in a mixed leukocyte culture reaction (MLC-stimulated) were harvested daily for 5 days and assessed for MIF production. Maximal activity was present by $48 \mathrm{~h}$ and did not increase thereafter, through the fifth day, when all subsequent MLC-stimulated cultures were harvested.

Comparison of MIF activity of MLC-stimulated adult, cord, and newborn cells is shown in Fig. 6. MIF activity in supernatants from autologously stimulated adult (A), cord (C), and newborn (N) lymphocytes were $20 \pm 2 \%, 21 \pm 3 \%$, and $18 \pm 2 \%$, respectively. The MIF activity of undiluted supernatants from adult ( $n$ $=11)$, cord $(n=9)$, and newborn $(n=12)$ lymphocytes stimulated by pooled allogeneic irradiated cells showed significant differences 


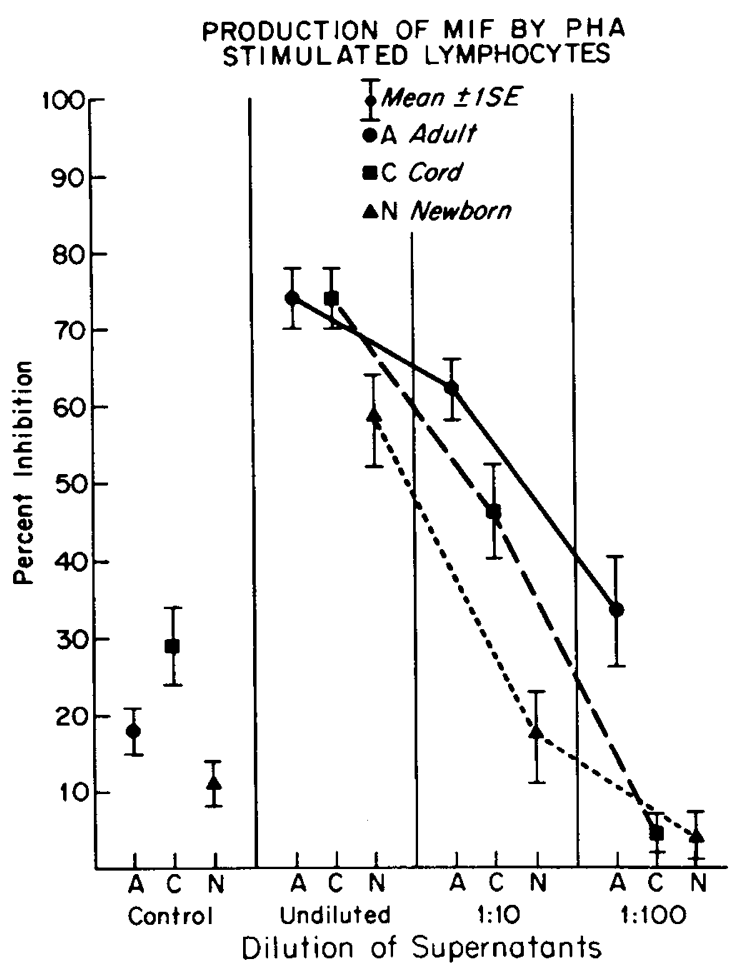

Fig. 5. Migration inhibition factor (MIF) production of adult, cord, and newborn lymphocyte preparation after activation with phytohemagglutinin (PHA) for $72 \mathrm{~h}$. The MIF is measured as a \% inhibition of unstimulated supernatant migration. The first vertical column shows the mean ( \pm S.E.) MIF activity of the supernatants without PHA; the second column, the MIF activity of undiluted supernatants from the PHA-stimulated cultures; the third column, the 1:10 dilution of supernatants from the PHA cultures; and the fourth column, 1:100 dilution of supernatant from the PHA cultures. A comparison of MIF activity can be made by determining the supernatant dilution that gives $50 \%$ inhibition. This is achieved at a 1:30 dilution of adult supernatant, a 1:8 dilution of newborn supernatant, and a 1:2 dilution of cord supernatant, indicating that cord blood lymphocytes and newborn lymphocytes have about $25 \%$ and $8 \%$, respectively of the MIF activity of adult lymphocytes, a significant difference.

of $64 \pm 2 \%, 26 \pm 6 \%$, or $35 \pm 3 \%$, respectively. When newborn or cord supernatants were diluted 1:50, MIF activity was no longer detectable in significant amounts, whereas, adult supernatants at this dilution still had MIF activity. A comparison of the dilutions that would produce $50 \%$ activity with MLC-stimulation indicates that cord lymphocytes have $13 \%$ and newborn lymphocytes have $2 \%$ of the MIF activity from adult lymphocytes.

Stimulation indices for the MLC cultures showed no significant difference between newborn and adult cells. Because the autologously stimulated (resting) cord lymphocytes have a higher rate of spontaneous activity $(510 \pm 100)$, stimulation indices for the MLC cord cultures $(17 \pm 3)$ were significantly less than those of either adult $(30 \pm 5)$ or newborn $(39 \pm 7)$ leukocytes.

PHA-stimulated LIF production. The 48-h supernatants from PHA-activated lymphocyte culture from 10 adults and eight cord samples were assayed for LIF activity. The LIF activity of undiluted samples from adult or cord lymphocytes was $62.3 \pm 19.7 \%$ and $45.0 \pm 22.3 \%$, respectively $(P>0.05)$. When diluted, LIF from cord samples was absent at a dilution of 1:10, whereas minimal activity remained from adult derived cultures. Comparison of the dilutions at which $50 \%$ inhibition occurred demonstrated that PHA-induced LIF production in cord blood was $27 \%$ of adult levels.

Comparison of LIF and MIF in adult and cord samples. LIF and MIF activity were assayed simultaneously in supernatants from PHA-stimulated adult or cord blood lymphocytes. In the adult, similar activities existed for both lymphokines at undiluted and 1:10 dilution. At 1:100 dilution, significantly more LIF activity was noted $(P<0.01)$ (see Fig. 7). In cord samples, the converse was noted. LIF activity was significantly less than MIF activity at undiluted $(P=0.02)$ and $1: 10$ dilution $(P<0.01)$ (Fig. 7). This indicates relatively more MIF in adults and relatively more LIF in cord bloods.

\section{DISCUSSION}

Although the term lymphokine refers to substances produced by lymphocytes, monocytes or specific lymphocyte subsets may be involved in their synthesis. Deficient synthesis of lymphokines in cord lymphocytes was reported by Eife, et al. (7) using a cytotoxic assay against a human hepatoma target cell. Some of the newborns exhibited lymphotoxin production equivalent to the lowest adult controls, demonstrating that the activity was not completely absent. In a previous study of MIF production by cord blood lymphocytes Muller et al. (15) using guinea pig rather than human macrophages, noted that five of 17 cultures of cord blood lymphocytes, which were stimulated with PHA, produced detectable quantities of MIF compared to 8 of 14 adult lymphocyte preparations. In this system, $80 \%$ migration was the upper limit for significant inhibition of migration. There was more inhibition (mean $79 \%$ ) by adults than in infants $(97 \%$ ) but the mean inhibition did not differ significantly. After stimulation of cord lymphocytes by soluble Concanavalin $\mathrm{A}$, insoluble Concanavalin $\mathrm{A}$, and PPD the mean migration inhibition was 90,71 , and $130 \%$, respectively. Because mean MIF activity to the three mitogens was not

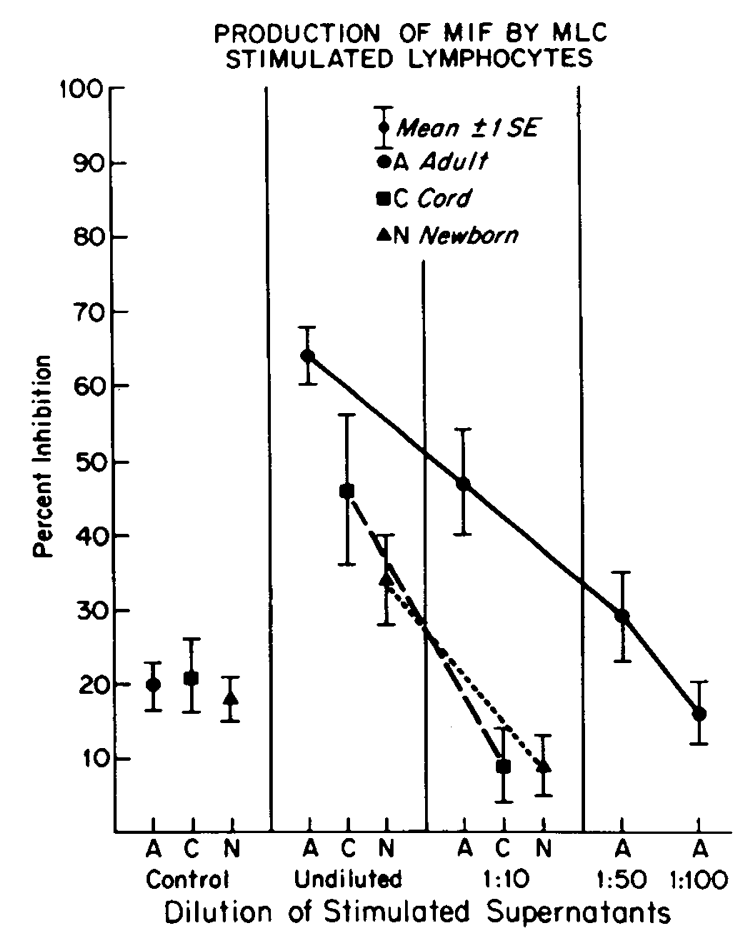

Fig. 6. Migration inhibition factor (MIF) production of 20 adult, 18 cord blood, and 17 newborn lymphocyte preparations after culture with irradiated allogeneic lymphocytes in a mixed leukocyte culture (MLC) for 5 days. The MIF activity is measured as a \% inhibition of unstimulated supernatant migration. The first vertical column shows the mean ( \pm S.E.) MIF activity of the supernatants without allogeneic (MLC) lymphocytes; the second column, the undiluted supernatants from the MLC cultures; the third column, the 1:10 diluted supernatants; and the fourth column, the 1:50 or : 100 diluted supernatants. A comparison of MIF activity, made by determining the supernatant dilution that gives $50 \%$ inhibition, indicates that cord and newborn lymphocytes have about $10 \%$ and $5 \%$, respectively, the MIF activity of adult lymphocytes, a significant difference. 


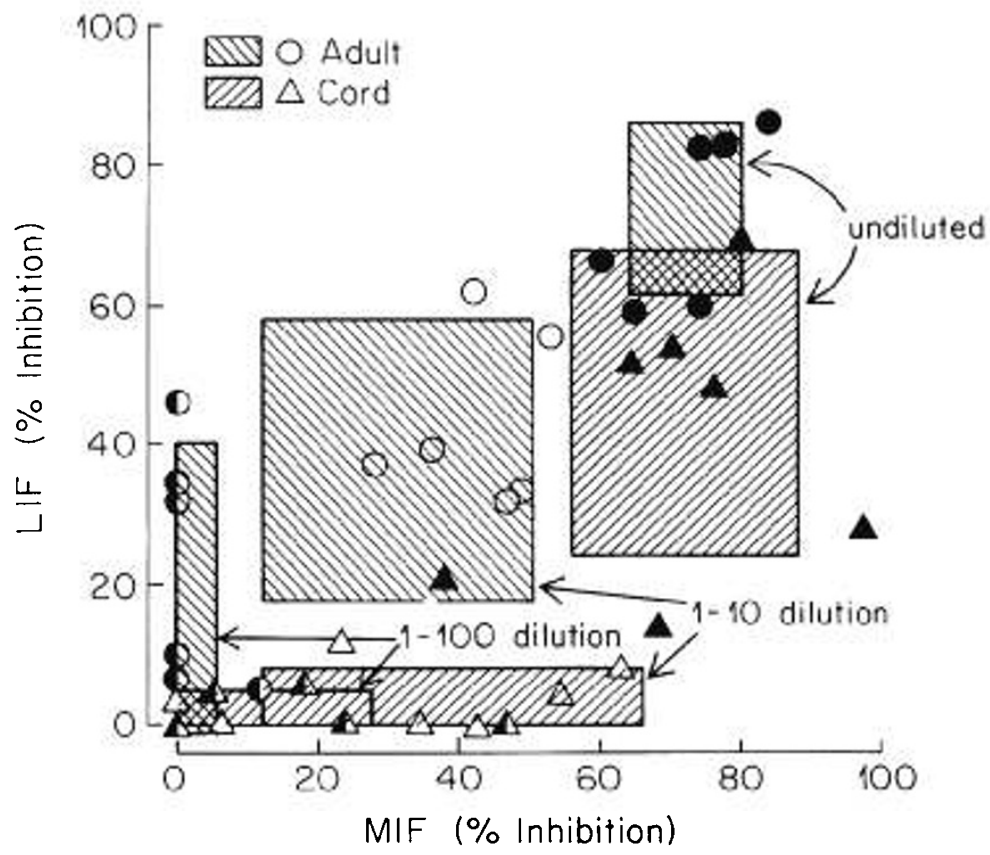

Fig. 7. Comparison of migration inhibition factor (MIF) and leukocyte inhibition factor (LIF) activity and cord blood lymphocytes at three different strengths (undiluted, $1: 10,1: 100$ ). The six boxes embrace \pm 1 S.D. of both MIF and LIF activity at each dilution for cord and adult bloods. In undiluted samples (solid symbols) both activities were approximately equivalent. At 1:10 dilution (open symbols), cord bloods have more MIF activity than LIF activity. At 1:100 dilutions (semi-solid symbols), adult bloods have relatively more LIF activity than MIF activity.

significantly different between adult and newborn, the authors concluded there was no defect in MIF production in cord lymphocytes. Close inspection of their data shows that significant MIF production (<80\% inhibition) occurred in 15 of $35(45 \%)$ cord blood mitogen activated cultures. In four of $35(11 \%)$ cultures there were high amounts ( $<60 \%$ inhibition) of MIF produced. This is in accord with our data which show deficient but not absent MIF production after PHA-stimulation. The quantitative differences in MIF or LIF production can only be appreciated by performing lymphokine assays on dilutions of supernatants as performed with our method.

Several studies support the concept that lymphocytes from normal newborns can synthesize adult quantities of some lymphokines, e.g., classical interferon, monocyte chemotactic factor, but not others (e.g., immune interferon, lymphotoxin) (3). Studies in MIF and LIF in newborns show variable results. Hahn et al. (9) found LIF to be normal in cord blood and observed that although the synthesis of LIF in normal cord blood leukocytes after activation with PHA was equivalent to that synthesized by adult cells, newborns with Trisomy 21 had defective LIF production. We found that the amount of LIF in the supernatant of PHA-activated cord leukocytes produced inhibition equivalent to supernatants from PHA-activated adult lymphocytes. This activity could be diluted out rapidly, indicating a deficiency of $27 \%$ of adult synthesis.

Comparison between LIF and MIF activity in PHA activated cord and adult samples revealed an unsuspected discrepancy. In adult supernatants, MIF activity could be diluted out more readily than LIF activity; in cord supernatants, LIF activity could be diluted out more readily than MIF activity. This supports the observation that LIF and MIF are distinct (3) and suggests that MIF derived after PHA-stimulation may be produced in greater quantities or in a more potent form by cord lymphocytes when compared with that of adult lymphocytes. LIF and MIF are related but distinct lymphokines (13).

These observations may have important clinical relevance as the inflammatory response of newborns may be associated with deficient mediator activities of attracting, holding, and stimulating polymorphonuclear cells and macrophages at a site of local infection.
The newborns' selective lymphokine deficiency may be similar to that of patients with certain immunodeficiencies, specifically deficient immune interferon production in selective IgA deficiency (8), deficient MIF production and cutaneous anergy in chronic mucocutaneous candidiasis (18) and deficient classical interferon synthesis in protein-calorie malnutrition (19).

\section{REFERENCES AND NOTES}

1. Andersson, T., Stejskal, V., and Harfast, B.: An in vitro method for study of human lymphocyte cytotoxicity against mumps-virus-infected target cells. J. Immunol., 114: 237 (1974)

2. Bonforte, R. J., Topilsky, M., Siltzbach, L. E., and Glade, P. R.: Phytohemagglutinin skin test: A possible in vivo measure of cell-mediated immunity. J. Pediatr. 81: 775 (1972).

3. Bryson, Y. J., Winter, H. S., Gard, S. E., Fischer, T. J., and Stiehm, E. R. Deficiency of immune interferon production by leukocytes of normal newborns. Cell. Immunol., 55: 191 (1980).

4. Buetow, K. C., Klein, S. W., and Lane, R. B.: Septicemia in premature infants. Am. J. Dis. Child. 110: 29 (1965).

5. Campbell, A. C., Waller, C., Wood, J., Aynsky-Green, A., and Yu, V.: Lymphocyte subpopulation in the blood of newborn infants. Clin. Exp. Immunol., 18: 469 (1974).

6. Cutler, J. E.: A simple in vitro method for studies on chemotaxis. Proc. Soc. Exp Biol. Med., 147: 471 (1974).

7. Eife, R. F., Eife, G., August, C. S., Kuhre, W. L., and Staehr-Johansen, K. Lymphotoxin production and blast cell transformation by cord blood lymphocytes: Dissociated lymphocyte function in newborn infants. Cell. Immunol. 14: 435 (1974)

8. Epstein, L. B. and Ammann, A. J.: Evaluation of T lymphocyte effector function in immunodeficiency diseases: abnormality in mitogen-stimulated interferon in patients with selective IgA deficiency. J. Immunol., 112: 617 (1974).

9. Hahn, T., Levin, S., and Handzel, Z. T.: Leucocyte migration inhibition factor (LIF) production by lymphocytes of normal children, newborns, and children with immune deficiency. Clin. Exp. Immunol., 24: 448 (1976).

10. Klein, R. B., Fischer, T. J., Gard, G. E., Biberstein, M., Rich, K. C., and Stiehm, E. R.: Decreased mononuclear and polymorphonuclear chemotaxis in human newborns, infants, and young children. Pediatrics, 60: 467 (1977).

11. Leiken, S., Mochir-Fatemi, F., and Park, K.: Blast transformation of lymphocytes from newborn human infants. J. Pediatr., 72: 510 (1968).

12. Leikin, S., Whang-Peng, J., and Oppenheim, J. J.: Proc. of the Fifth Leukocyte Culture Conference. pp. 389-402 (Academic Press, New York, 1970)

13. Lomnitzer, R., Robson, A. R., and Koornhof, H. J.: Production of leucocyte inhibitory factor (LIF) and macrophage inhibitory factor (MIF) by PHAstimulated lymphocytes. Clin. Exp. Immunol., 22: 522 (1975).

14. McConnachie, P. M., Rachelefsky, G., Stiehm, E. R., and Terasaki, P. I.: Antibody-dependent lymphocyte killer function and age. Pediatrics, 52: 795 (1973). 
15. Muller, M. R., Lazary, S., and Hitzig, W. H.: Production of migration inhibitory factor and blast cell transformation by cord blood lymphocytes. Immunology, 50: 593 (1976).

16. Nelson, R. D., Quie, P. G., and Simmons, R. L.: Chemotaxis under agarose: new and simple method for measuring chemotaxis and spontaneous migration of human polymorphonuclear leukocytes and monocytes. J. Immunol., 115 1650 (1975).

17. Olding, L.: Bacterial infection in cases of perinatal death: A morphologic and bacterial study based on 264 autopsies. Acta Pediatr. Scand. Supp., 171: (1966)

18. Rocklin, R. E., Chilgren, R. A., Hong, R., and David, J. R.: Transfer of cellula hypersensitivity in chronic mucocutaneous Candidiasis monitored in vivo and in vitro. Cell Immunol., 1: 290 (1970).

19. Schlesinger, L., Ohlbaum, A., Grez, L., and Stekel, A.: Malnutrition and the Immune Response. R. M. Suskind, Ed. pp. 91-98 (Raven Press, New York, 1977).

20. Sengar, D. P. S. and Terasaki, P.: A semimicro mixed leukocyte culture test. Transplant, 11: 160 (1971)

21. Shore, S. L., Milgrom, H., Wood, P. A., and Nahmias, A. J.: Antibody-dependent cellular cytotoxicity to target cells infected with herpes simplex viruses: functional adequacy in the neonate. Pediatrics, 59: 22 (1977).

Copyright (c) 1983 International Pediatric Research Foundation, Inc $0031-3998 / 83 / 1707-0573 \$ 02.00 / 0$
22. Stites, D. P., Wybran, J., Carr, M. C., and Fudenberg, H. H.: Ontogeny of Acquired Immunity. Ciba Foundation Symposium, pp 113-139 (Elsevier, Amsterdam, 1977)

23. Toivanan, P., Granberg, G., and Manninen, K.: Regulatory Mechanisms in Lymphocyte Activation, edited by D. O. Lucas, pp 638-640 (Academic Press, New York, 1970).

24. Uhr, J., Dancis, J, and Neumann, C. G.: Delayed-type hypersensitivity in premature neonatal humans. Nature, 187: 1130 (1960)

25. We wish to thank Ms. Shirley Lerner and Ms. Winifred Cole for technical assistance, and the Nursing Service for their cooperation in obtaining the samples.

26. Present address: Children's Hospital Medical Center, 300 Longwood Avenue, Boston, Massachusetts 02115 .

27. Present address: Children's Hospital, Cincinnati, Ohio.

28. Requests for reprints should be addressed to: Dr. E. R. Stiehm, UCLA Center for the Health Sciences, Los Angeles, California 90024

29. Supported in part by Grants from the U.S. Public Health Service (HD-09800), National Foundation (6-19), and National Institutes of Health New Investigator Research Award (1-R23-AM-31070).

30. Received for publication April 22, 1982.

31. Accepted for publication November 15, 1982. 